alcoholic liver disease than in healthy people. Thus dextropropoxyphene is not a recommended analgesic for alcoholics. When the drug alone has been taken in excessive doses in other patients saturation of the first-pass metabolism may take place. ${ }^{4}$

The authors further suggest that the dangers of dextropropoxyphene outweigh the analgesic activity of the drug. They cite Miller's review of the drug ${ }^{5}$ in support of their argument. However, in their introduction mention is made of the fact that Distalgesic is by far the most commonly prescribed drug. Recently Dr C I T Cruickshank (7 June, p 1378) suggested that Distalgesic was the drug of first choice for analgesia among his consultant colleagues. To us it would appear clear that the analgesic efficacy of the drug is responsible for its widespread use. Dextropropoxyphene finds its greatest use in musculoskeletal disorders and as an adjunct in rheumatoid arthritis; only a few trials dealing with these are found in Miller's reviews. ${ }^{56}$ Moreover, many of the papers reviewed by Miller were single-dose studies, whereas studies indicate greater analgesic activity in multiple doses.

Dextropropoxyphene addiction has been reported in Britain by Whittington ${ }^{8}$ and in a recent short report by Dr Robert Wall and others (17 May, p 1213). The two patients concerned were, however, taking 27 and 32 Distalgesic tablets daily respectively (recommended maximum dose eight tablets per day). Moreover, there was a previous history of abuse of other drugs. We have found no reports of addiction to dextropropoxyphene at recommended therapeutic doses. A preliminary survey of rheumatoid outpatients ${ }^{9}$ showed no evidence of tolerance or addiction to Distalgesic.

The emotive issue of fatalities where dextropropoxyphene is said to be implicated is certainly a case for dispassionate and objective investigation and further research. Current reports, important as they are, which highlight the self-poisoning issue do tend to conceal the fact that probably a million or more patients in the United Kingdom alone safely take the drug for effective relief of pain.

P S DWYER I F JONES

Postgraduate School of Studies in Pharmacy,
Bradford, W Yorks BD7 1DP

1 Fraser DM, Lawson AAH. Health Bull 1975;33:1.

2 Lawson AAH, et al. Health Bull 1979;37:121-7.1.
3 Perrier D, Gibaldi M. I Clin Pharmacol 1972;12: Perrier D.
449-52.

449-52.
Schou J, et al. Arch Toxicol 1978; suppl 1:343-6.

'Miller RR, et al. FAMA 1970;213:996-1006.

? Miller RR. Am F Hosp Pharm 1977;34:413-23.

8 Waife SP, et al. Int f Clin Pharmacol 1975

- Farrell J, Brown AW, Sturrock RD. Br Med f 1979. i:1284.

\section{Lithium and the kidney}

SIR, - In an intensive investigation ${ }^{1}$ on renal function in 101 patients treated with lithium for periods ranging from 1 to $12 \frac{1}{2}$ years we found, like Drs N J Birch and R P Hullin (3 May, p 1148), little evidence of serious impairment of renal function attributable to lithium therapy. Creatinine clearance, serum creatinine, maximum urine osmolality after desmopressin (DDAVP) administration, urine and plasma $\beta_{2}$-microglobulin, and the urinary excretion of $N$-acetyl- $\beta$-glucosaminidase were not significantly different between patients on lithium and depressed patients never treated with lithium. Only $1 \%$ had a urine volume greater than 3.5 litres.

Our dosage regimen, however, was different to that used by Drs Birch and Hullin. Our patients were treated with lithium carbonate (Priadel), given once a day in late evening to give a mean plasma level of $0.86 \mathrm{mmol} / 1$ $(0.59 \mathrm{mg} / 100 \mathrm{ml}) 12$ hours after the last dose (the mean daily lithium dosage was 23.5 $0.8 \mathrm{mmol}(16.2 \pm 0.55 \mathrm{mg} / 100 \mathrm{ml})$ for women and $30.9+1.0 \mathrm{mmol}(21.32+0.69 \mathrm{mg} / 100 \mathrm{ml})$ for men). We agree with Drs Birch and Hullin that the optimum prophylactic level may wel be lower than those previously advanced but we would suggest caution before reducing 12-hour serum levels to $0.4 \mathrm{mmol} / 1(0.28 \mathrm{mg}$ / $100 \mathrm{ml}$ ) until we have further evidence.

The data indicate that our regimen is safe, provides effective prophylaxis, is convenient for the patient, and ensures good compliance. MRC Neuropsychiatry Laboratory,
West Park Hospital,

AIEc Coppen Epsom, Surrey

\section{Department of Nephrology,} St Bartholomew's Hospital,
London EC1A 7BE ${ }^{1}$ Coppen A, Bishop M, Bailey J, Cattell WR, Price RG.
Acta Psychiat Scand (in press).

\section{Progesterone, fluid, and electrolytes in premenstrual syndrome}

SIR,-In all my writings and lectures on premenstrual syndrome I find it is essential to begin with a clear definition of the syndrome to avoid confusion with menstrual distress and because progesterone therapy is only effective in correctly diagnosed cases of premenstrual syndrome.

Mr P M S O'Brien and his colleagues (10 May, $p$ 1161) give no definition and it appears that they are confusing it with menstrual distress, as other authors have done recently. ${ }^{1}$ Premenstrual syndrome is defined as "symptoms which recur regularly in the same phase of each menstrual cycle, followed by a symptom-free phase in each cycle." 2 A minimum of seven days free from symptoms is required, although many women experience two or three weeks each cycle free from symptoms. Thus the diagnosis depends on the timing of the symptoms rather than the mere presence of characteristic symptoms or their duration. Symptoms which increase during the premenstrual week but are present throughout the cycle come under the diagnosis of "menstrual distress," not premenstrual syndrome. Many, if not most, women afflicted with endogenous depression and anxiety neurosis experience a premenstrual exacerbation; this is not premenstrual syndrome. Approximately half of all newly referred patients attending the premenstrual syndrome clinic at University College Hospital, although presenting with characteristic symptoms, are found not to be suffering from premenstrual syndrome according to the above quoted definition when their symptoms are properly charted.

There is no relationship between the severity and duration of symptoms present in premenstrual syndrome. The explosion of symptoms in cases of epilepsy, baby battering, and overdose attempts may last only a day or two and may be unaccompanied by bloatedness or alteration in libido and so would merit a low score on the daily change of mood scores used by $\mathrm{Mr}$ O'Brien and his colleagues.

No mention is made in the paper of the ages, parity, or social class of the subjects, although one may assume that they possessed a fair intelligence to enable them to differentiate on a daily linear scale depression from sadness and anxiety from tension. Libido may alter, in both the positive and the negative direction, in premenstrual syndrome and change of libido can hardly be considered characteristic of the syndrome; but no indication is given of whether increased or decreased libido rated higher on the visual analogue scales.

Endocrinologists are increasingly recognising that it is the free proportion, as opposed to the bound fraction, of the hormone which is of clinical significance. Mr O'Brien and his colleagues have reached their conclusion from a study of measurements of total serum progesterone without waiting for reliable estimations of free progesterone.

Ever since $\mathrm{Dr}$ Raymond Greene and I published the first paper in 1953 on the use of progesterone injections for the treatment of premenstrual syndrome, with a success rate of $83 \%,^{3}$ a steadily increasing stream of correctly diagnosed patients has been successfully treated with progesterone pessaries and suppositories as well as by injection. This has proved to be the most successful form of treatment for premenstrual syndrome. Menstrual distress, however, is a completely different problem.

K Dalton

London W1N $1 \mathrm{AE}$

1 Sampson G. Br f Psychiatry 1979;135:209-14. 2 Dalton $\mathrm{K}$. The premenstrual syndrome and progesterone therapy. London: Heinemann Medical Books, 1977

\section{Anorexia nervosa in diabetes mellitus}

SIR,-We were interested in the report by Drs C G Fairburn and J M Steel (10 May, p 1167), although their third case would not meet our criteria for anorexia nervosa. ${ }^{1}$ They do not state the size of their diabetic population, but since the prevalence of anorexia nervosa is about one in 100 in the most vulnerable group-that is, girls of $11-18$ in schools of good academic standing-the number of cases reported is probably that to be expected in a large diabetic clinic. The prevalence of insulin-dependent diabetes in the general population of the United Kingdom is about 1 in $400^{2}$ and is probably slightly higher in the age range at risk for anorexia nervosa, 11-35 years. Our series of 400 anorexics includes two with insulin-dependent diabetes, both of whom had a history of longstanding personality problems, more characteristic of secondary than of primary anorexia nervosa, and used vomiting (and laxatives) as well as dieting to control weight.

Our first patient developed anorexia nervosa at 16 , and presented in diabetic coma four years later. At 29 , this girl remains $15 \%$ underweight, amenorrhoeic, depressed, and unable to break away from her mother. She manages her diabetes competently, and this does not seem a major factor in her restricted life style. The two disorders in this case are coincidental. By contrast, in the other patient diabetes appears to have been a partial determinant of anorexia nervosa. She developed diabetes at nine; it interfered with school activities, to her distress, and family attitudes towards her altered. At 13, after menarche, when she weighed $56 \mathrm{~kg}$, she took a small overdose; 18 months later she developed anorexia 